\title{
Experimental tuna farming in the sultanate of OMAN
}

\begin{abstract}
Tuna are among a very large group of large and medium-oceanic fish and they are an important food source globally. They are also unusual fish because they are warm blooded fish and they need large amounts of oxygen. The fluctuations in tuna catch and price has led to focus on the farming of tuna in large floating cages in many countries, like South Australia, Turkey, Croatia, Mexico, Panama and other countries mainly for the Japanese market. In the Mediterranean they farm blue fin tuna, yellow fin tuna and big-eye are farmed in Mexico and the yellow fin tuna have been farmed on a pilot scale in the Sultanate of Oman by cage Culture Company.
\end{abstract}

To farm tuna several stages have to be practiced, starting from preparation of cages, fishing, transferring of the live fish to the towing cages and to the farming site, feeding, harvesting, processing, packaging and exportation.

In Oman, two types of cages were used in this process, towing cages and fattening cages, and with nets of 30 meters depth, fixed on cages of 48 meters diameter. Fishing was practiced using Purse-Seineships in areas around 50-70 kilometers away from the farming sites, nets were kept open to avoid killing of fish, which transferred under water to the towing cages then to the fattening cages. The speed of ship didn't exceed 4 nots/hour through the whole journey.

Tuna arrives to the farming site in a state of stress and seize eating for up to 3 weeks even if an attempt is made to feed them. After this period, tuna start feeding greedily large quantities of food with high oils content such as Sardine. When caught fish weight ranges from $25-40 \mathrm{KG}$, and there is no need to gain more weight but to increase the percentage of fat. In the Sultanate we used sardines, as the Omani sardine is characterized by high oil content. Feeding was distributed manually to the cage until the tuna stop eating. The fish lose about $20 \%$ of its weight during transportation and fasting stage, then compensate the loss and increase the percentage of oil in the flesh. Generally the universal calculated food conversion ratio is 1-20 as the fresh fish used in feeding has high proportion of water.

Harvesting process is a delicate process and should be organized well and requires accuracy and speed at work, relies on the use special collection net to collects almost the required number and detain it in the cage. Professional divers have to dive and swim with a hold of fish. The caught tuna then lifted from the waterslide on the smooth floating portion to reduce friction with the fish's body. Followed by quick killing of fish by putting steel nail in the brains of the fish to reduce stress and avoid burning of the meat. The second step is the insert of steel wire in the spinal cord after cutting the head using an electrics a win order to ensure the full death of the fish. Fish are then placed in ice water to ensure cooling of the meat and quality; the last stage is to transfer the fish to the factory for processing and exportation. Normally harvesting should not take more than 2-3 minutes to keep the meet to the highest quality or Shesimi grade for the Japanese market.

Dealing with farmed tuna inside the processing plant should be in a way that maintains fish quality and maintain me at temperature internally and externally and determine the quality of meat in terms of the percentage of the fats in fish flesh.
Volume 4 Issue 3 - 2016

Adel N Z Gindy

Department of Marine Science and Fisheries, A'Sharqiyah University, Oman

Correspondence: Adel N Z Gindy, Head of Department of Marine Science and Fisheries, A'Sharqiyah University, Ibra, Sultanate of Oman Email amitranjanfcri@gmail.com

Received: July 23, 2015 | Published: May 10, 2016

\section{Introduction}

Tuna is among a very large group of large and medium-oceanic fish and they are an important food source globally. They are also unusual fish because they are warm blooded and need large amounts of oxygen. The fluctuations in tuna catch and price has led to focus on the farming of tunain large floating cages in many countries, like Australia, Turkey, Croatia, Mexico, Panama and other countries; mainly for the Japanese market. In the Mediterranean, farmed tuna include: Northern blue fin tuna [Thunnusthynnus] and the yellow fin tuna [Thunnusal barcares], while big-eye tuna [Thunnus obesus] are farmed in Mexico. The yellow fin tuna has been farmed on a pilot scale in the Sultanate of Oman by Cage Culture Company. Tuna farming locations are shown in Figure 1.

The aim of fishing tuna for farming is to catch and transfer the live tuna from fishing nets to the towing cage and towing them to the site of fattening. In Australia, for example, fishing is in one place then fish is transferred to fattening grounds at distances of up to 500-600 kilometers, while in the Mediterranean, fishing maybe in the nearby regions of Turkey, sometimes transferred for fattening in Croatia over long distances. To farm tuna, several stages have to be followed, starting from cage preparation, fishing, transferring of live fish to the towing cages and to the farming site, feeding, farming activities, harvesting, processing, packaging and export stage Figure $2 .{ }^{1}$ 

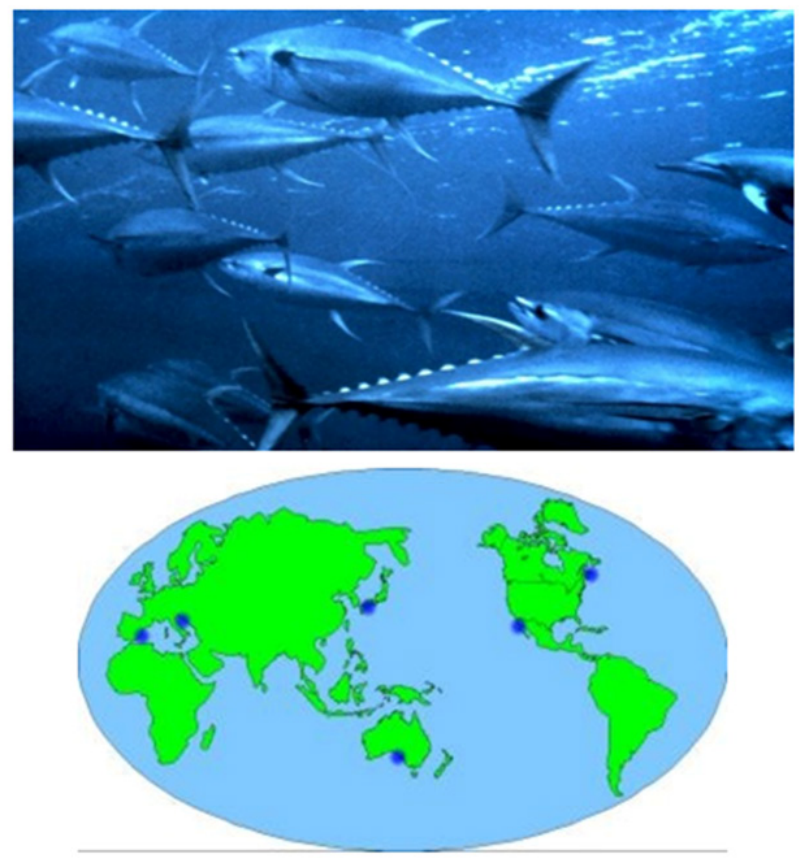

Figure I The major areas where tuna farming is practiced.

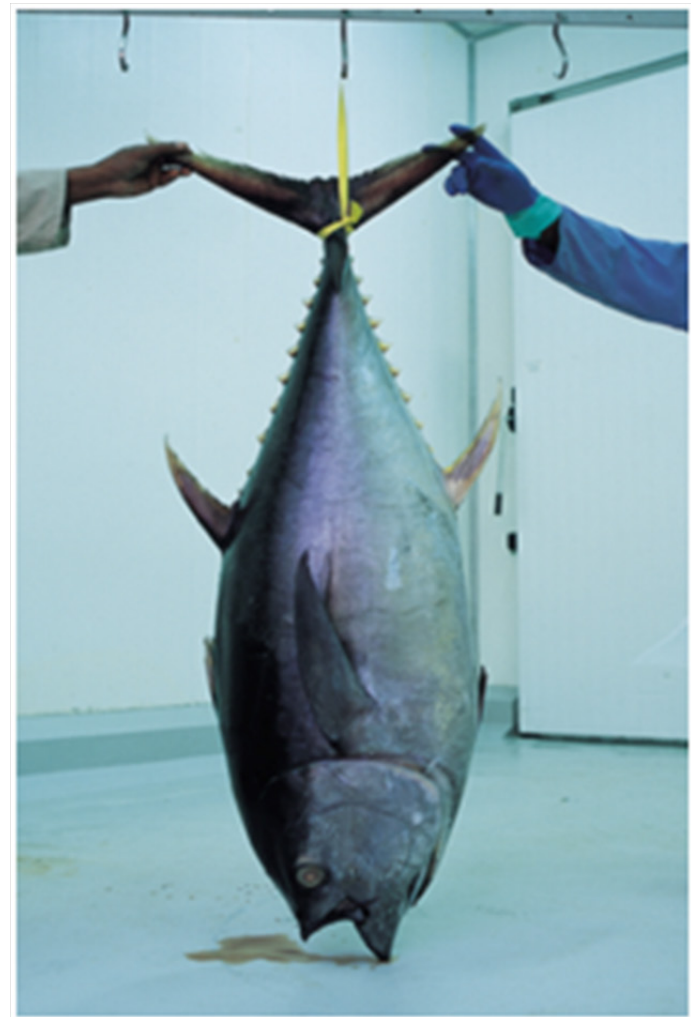

Figure 2 Yellow fin tuna (Thunnus albaracres).

There are two types of cages used in this process, towing cages and fattening cages. Towing cages are of the same quality of pipes [HDPVC] but of only one ring type, but the fattening cages are of two or three circular rings. The differences between the normal fish cages and the tuna cages are the size or diameter, strength and in particular the towing cages (Figure 3).

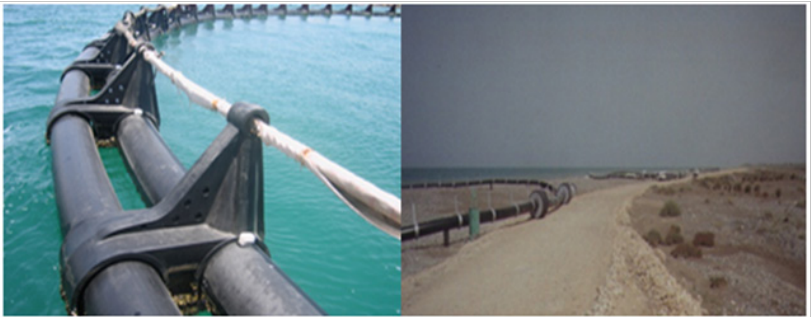

Figure 3 Fattening cage made of two rings and Towing cage for transferring the live tuna.The strong special links to withstand tuna from fishing net to the place of fattening the heavy work.

The next step is to fix the nets for both the towing cages and fattening cages, normally nets for the fattening cages have a depth of 30meters, installed to cages of 48 meters diameter therefore preparation of cages and installation of the nets before bringing fish from the fishing location to the fattening site has to be start several days before fish arrival.

When tuna fattening was practiced in the Sultanate of Oman, fishing was practiced in areas around 50-70 kilometers away from farming sits using Purse-Seine ships where the nets were kept open to avoid killing the fish before transferring under water to the towing cages and then to the fattening cages.

The aim of Purse-Seine ships for tuna fishing is keeping the tuna alive. The process of transferring the tuna from the fishing net, to the towing cages then to the farming site must be done by specialists. The cage has to be towed in a certain way while the ropes should fixed in a way to avoid cracking of pipes and allowing the escape of the fish and the speed of ship towing the cages should not exceed 4 nots/hour throughout the whole journey (Figure 4 \& 5).

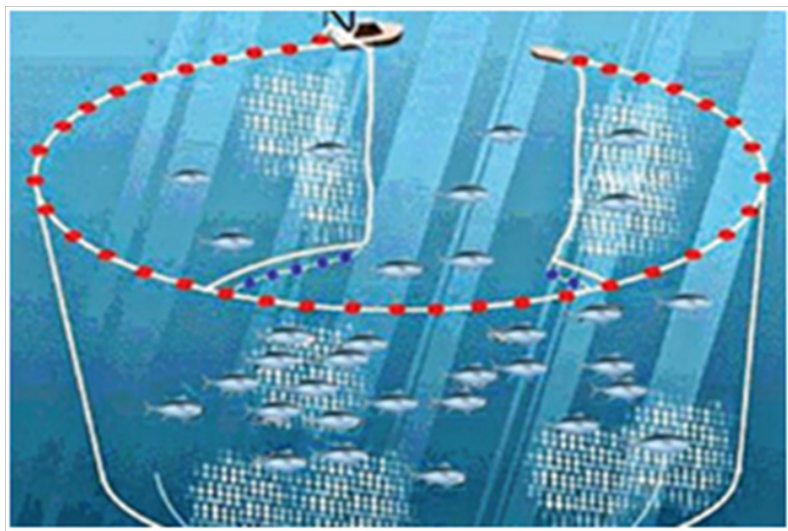

Figure 4 An open Purse-Seine fishing net and inside live fish, until transferred to the towing cage and to the fattening cages.

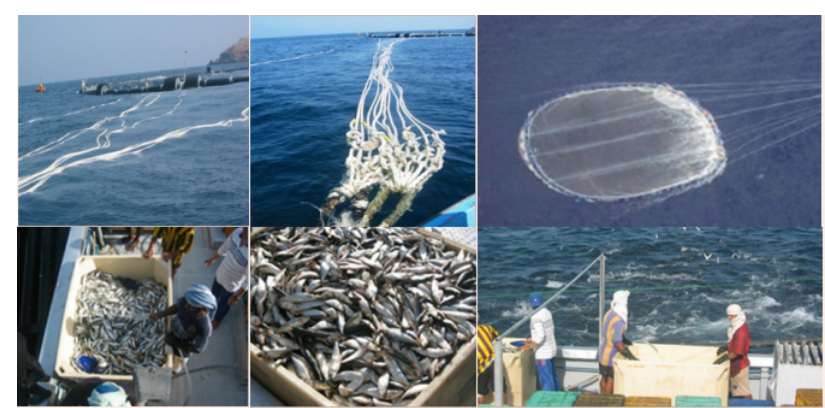

Figure 5 Explains how to set up the towing cages, notice the distribution of the ropes outside and inside the cage. 
After the arrival of the fish to the fattening site in a trip that can span between more than a months as happens in Australia to several days, as happened in the Sultanate of Oman with yellow fin tuna. In both cases, it arrives to the fattening site in a state of stress and stop eating for 2-3 weeks even if an attempt is made to feed it. After this period, tuna start feeding greedily on very large quantities of food which is high in natural oils such as Sardine and mackerel.

In farming the blue fin tuna in the Mediterranean feeding lasts for several months to increase the weight of the fish and increase the percentage of fat in fish flesh. In the Gulf region [Oman], fattening of the yellow find tuna practiced for a period of two to three months in order to raise the proportion of fat in the fish. The goal was not to increase fish weight because when caught fish weight ranges from $25-40 \mathrm{KG}$, which is the good weight for sale. However, there is no need to gain weight but to increase the proportion of fat to suite the Japanese market.

The process of Tuna fattening is performed using high oil content fish such as Anchovy, sardines and mackerel. When fattening of tuna in the Sultanate sardines were used as food for the tuna, as Omani sardines are characterized by high oil content. Feeding was practiced manually using fresh fish or frozen fish after defrosted and distributed regularly throughout the cage until the tuna stopped eating (Figure 6).

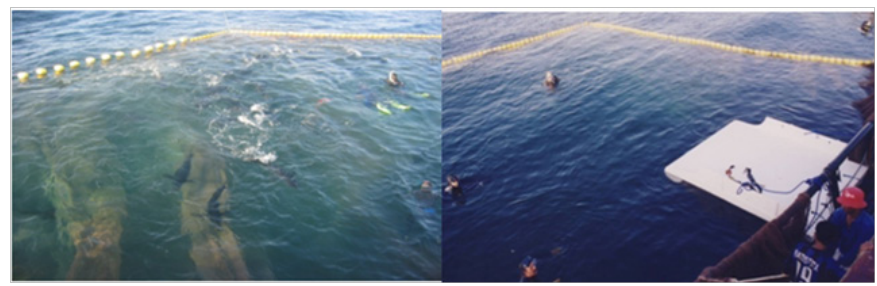

Figure $\mathbf{6}$ Large quantities of sardines that have been used in feeding Yellow-fin tuna in the Sultanate of Oman.

The food conversion rate is a complex process where the fish lose about $20 \%$ of it's weighing during transportation and fasting stage [3 weeks] and then follows compensation for the loss and then increase in the percentage of oil in the flesh. Generally the universal calculated food conversion ratio [FCR] is $1-20$ as the fresh fish that are used in feeding has a high percentage of water.

Harvesting process is a delicate activity, should be very well organized and requires accuracy and speed at work and relies on the use of special collection net (Figure 7) to collects almost the required number and detain it in the cage, followed by professional divers chasing the fish and getting hold of them, this process is a difficult process and needs effort and concentration; followed by the lifting of the fish from the water onto a smooth floating panel to reduce friction with the fish's body.

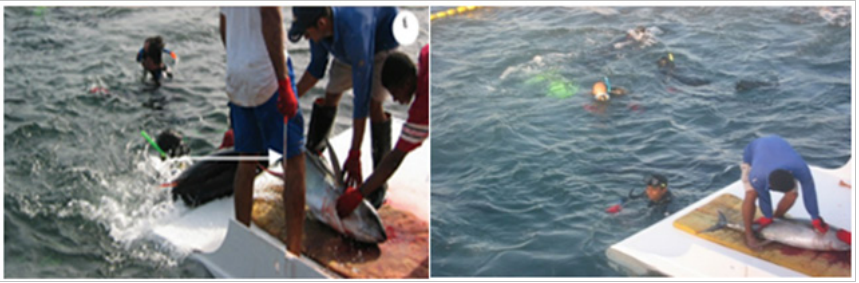

Figure 7 Drivers catching the yellow fintuna, arrow indicates the metal part which is used in the rapid killing of fish after removing from the water.

After lifting the tuna, the first step to kill the fish is by putting special steel nail in the brain of the fish to reduce stress and avoid burning of the meat. On the boat the second step is cutting the head using an electrics a wand insert of steel wire in the spinal cord in order to ensure the full death of the fish. Fish are then placed in ice water to ensure cooling of the meat and preserving meat quality. The last stage is to send the fish to the factory for processing and exportation (Figure 7-10).

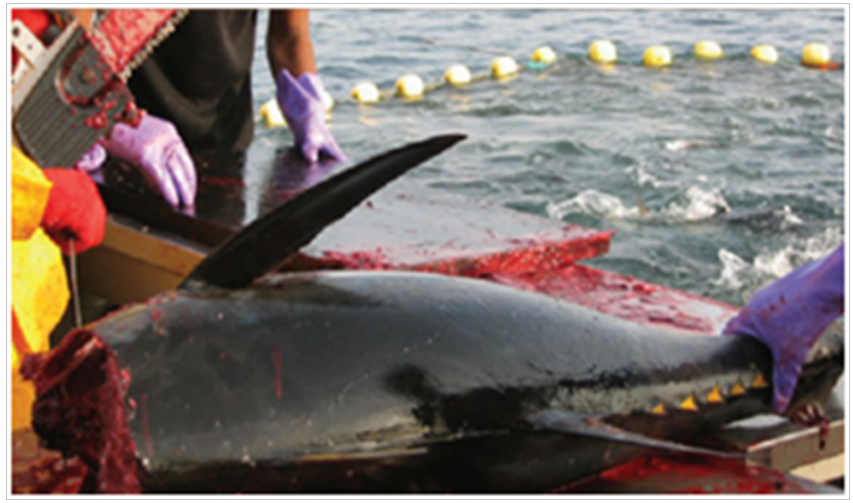

Figure 8 Cutting of head and evisceration immediately after, the fish out of the water.

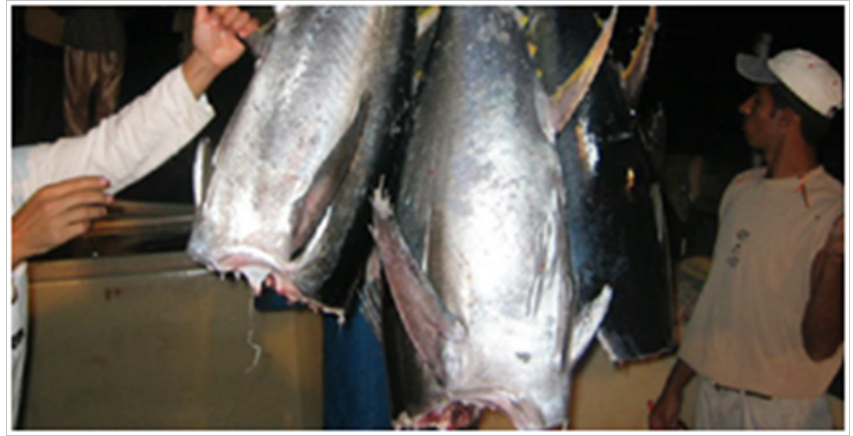

Figure 9 Group of yellow fintun a fish harvested from cage after cutting the head and evisceration just before putting in ice.

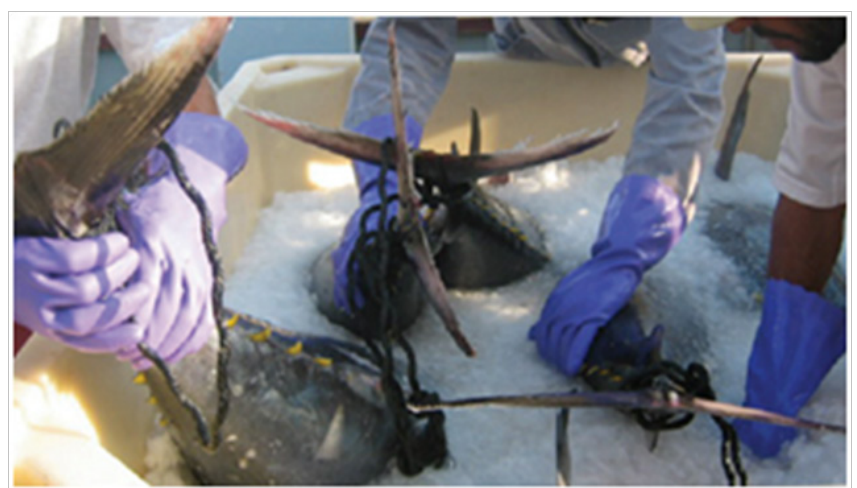

Figure 10 Fish placed in ice and water for transport to the packing plant.

Dealing with farmed tuna within the plant must be in a way that maintains the quality of the fish so as to obtain a higher price in exportation, which is often to the Japanese market. At the factory, several important operations take place to maintain theme at temperature internally and externally and take samples to determine the quality of meat in terms of the percentage of the oils in fish flesh. Within the processing plant, a set of several steps are taken during the processing and packaging of farmed tuna (Figure 11-17). 


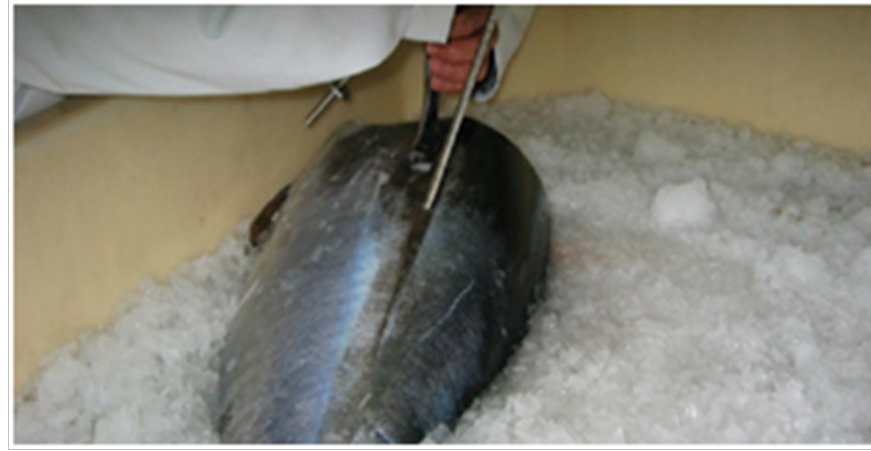

Figure I I Sample off armed tuna at the time of arrival to the factory for exportation chilled.

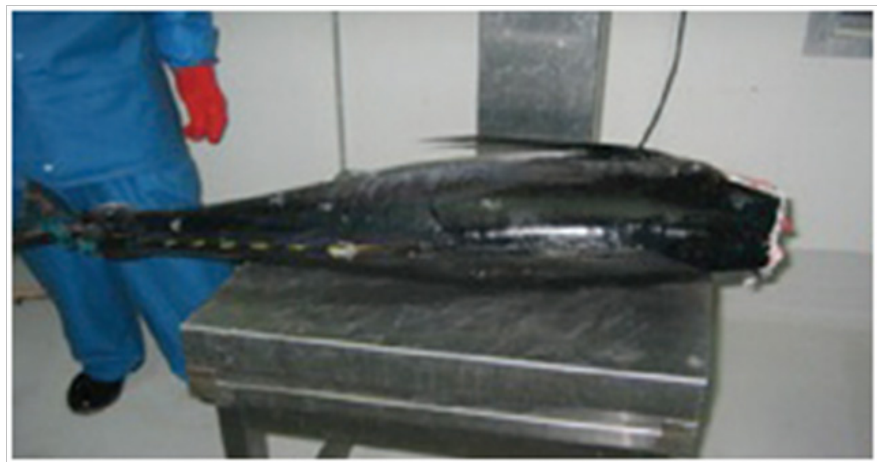

Figure 12 Weighing of a fish at the time of arrival to the factory for determination of the average of the weight the tuna batch.

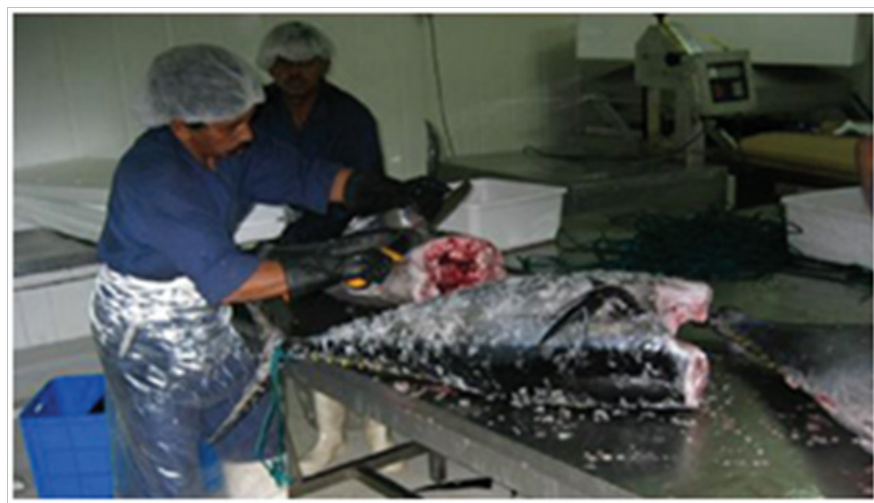

Figure I2. I Cleaning operations to ensure quality.

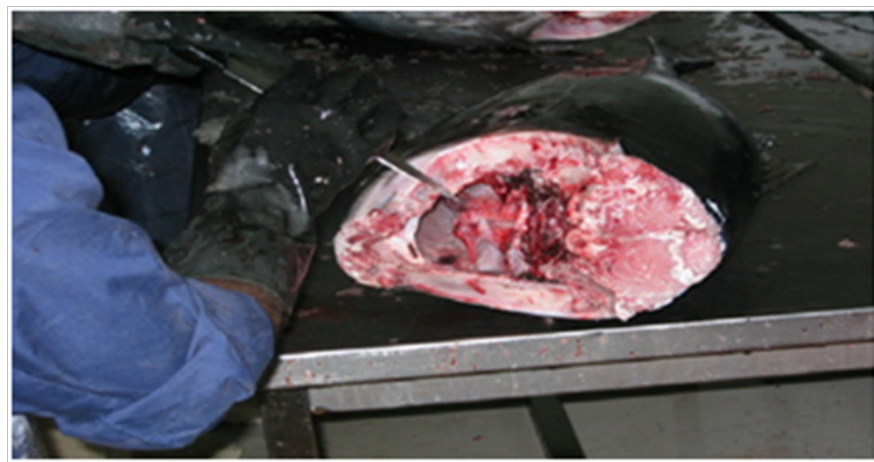

Figure I 2.2 Cleaning operations to ensure quality.

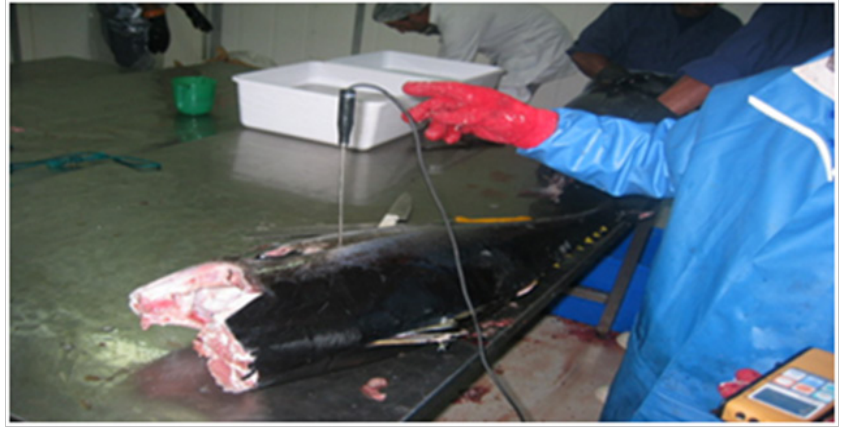

Figure 12.3 Measuring the internal temperature to indicate the degree of consistency of internal and external temperature, and the impact of meat temperature on meat quality (temperature should not get close to freezing point otherwise fish should be considered frozen).

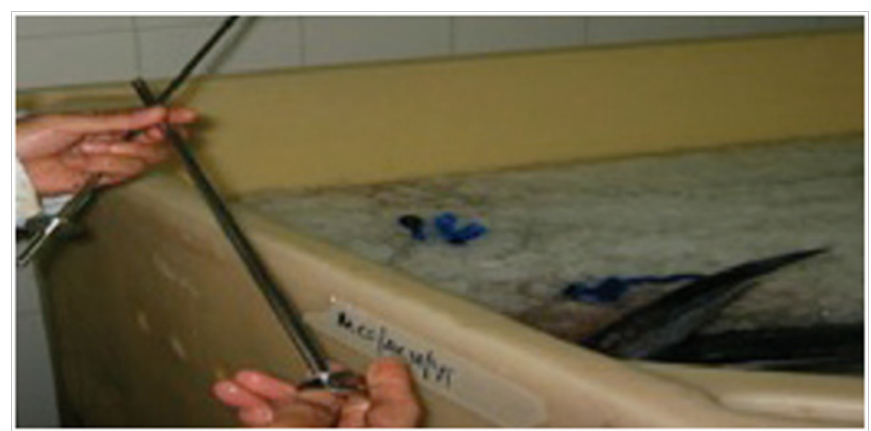

Figure 1 2.4 The process of taking a sample of fish flesh using as ample tube, the Sampling tube is a vacuum steel tube that enters the fish's body and Pulls the meat from the inside.

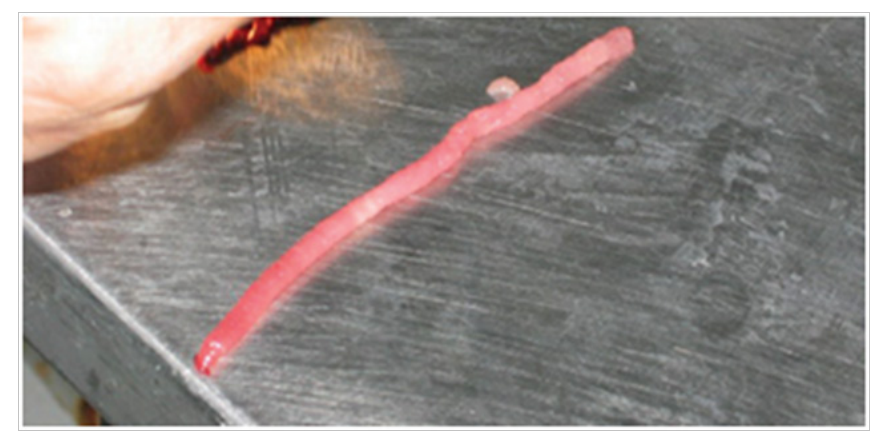

Figure I 3 Ample of tuna taken from inside the using sampling tube.

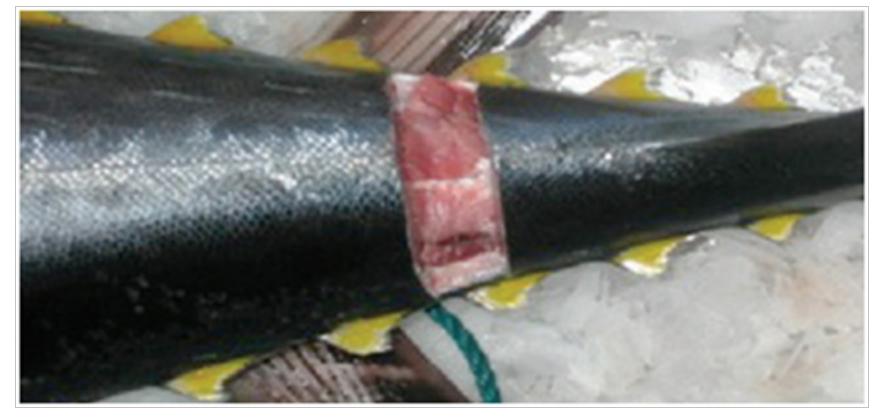

Figure I4 The other method of sampling is to cut a section close to the tail region, and to take for examination to determine the percentage off at in the meat. The percentage off at in the meat determines the price of exportation to the Japanese market therefore the stage of checking the percentage of at in the meat is an important stage of marketing the farmed tuna. 


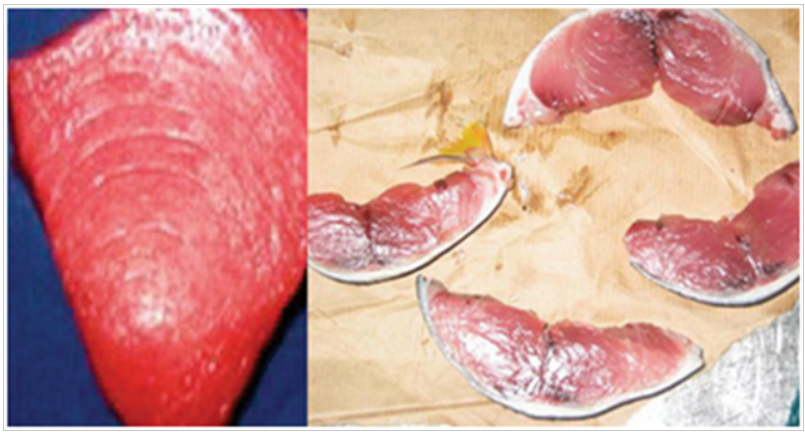

Figure 15 Shows the form of tuna meat fattened in cages that were fed foods High in the percentage of oils please observe gloss meat at the high proportion of oils.

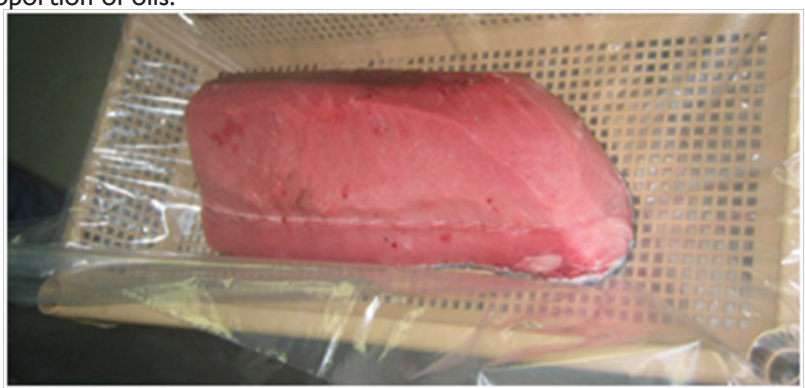

Figure 16 Tuna caught from the sea. Note with it has high quality meet but there is no glitter in the meat which shows the decline in the proportion of fat.

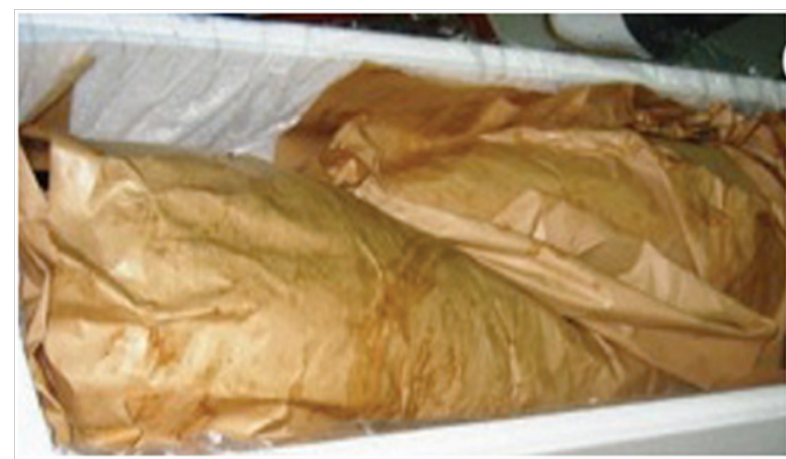

Figure 17 Farmed tuna fish wrapped securely; ice will cover the fish on top to keep the meat fresh in the case of export.
The price of exportation to the Japanese market Therefore the stage of checking the percentage of fat in the meat is an important stage of marketing the farmed tuna. ${ }^{2-6}$

\section{Acknowledgments}

None.

\section{Conflicts of interest}

None.

\section{References}

1. Floro Mercene Yellowfin tuna farming Tempo, News in Flash, Tuna. 2015

2. McAvoy A Hawaii regulators approve first US tuna farm. Associated Press, US. 2009

3. Jump up Cultivation, seedling production, and selective breeding of Bluefin tuna and other fish atthe Kinki University Fisheries Laboratory. 2010

4. http://factsanddetails.com/world/cat53/sub340/item2188.html

5. Sci-techfuturis. Breakthrough in Bluefin Tuna farming. European Commission euro news. 2015

6. Cladis DP, Kleiner AC, Freiser et al.Fatty Acid Profiles of Commercially Available Finfish Fillets in the United States. Lipids. 2014;49(10):10051018. 